\title{
SLIDING VECTOR FIELDS FOR NON-SMOOTH DYNAMICAL SYSTEMS HAVING INTERSECTING SWITCHING MANIFOLDS
}

\author{
JAUME LLIBRE $^{1}$, PAULO R. DA SILVA ${ }^{2}$ AND MARCO A. TEIXEIRA ${ }^{3}$
}

\begin{abstract}
We consider a differential equation $\dot{p}=X(p), \quad p \in \mathbb{R}^{3}$ with discontinuous right-hand side and discontinuities occurring on an algebraic variety $\Sigma$. We discuss the dynamics of the sliding mode which occurs when for any initial condition near $p \in \Sigma$ the corresponding solution trajectories are attracted to $\Sigma$. First we suppose that $\Sigma=$ $H^{-1}(0)$ where $H$ is a polynomial function and $0 \in \mathbb{R}$ is a regular value. In this case $\Sigma$ is locally diffeomorphic to the set $\mathcal{F}=\left\{(x, y, z) \in \mathbb{R}^{3} ; z=\right.$ $0\}$ (Filippov). Second we suppose that $\Sigma$ is the inverse image of a non-regular value. We focus our attention to the equations defined around singularities as described in [8]. More precisely, we restrict the degeneracy of the singularity so as to admit only those which appear when the regularity conditions in the definition of smooth surfaces of $\mathbb{R}^{3}$ in terms of implicit functions and immersions are broken in a stable manner. In this case $\Sigma$ is locally diffeomorphic to one of the following sets $\mathcal{D}=\left\{(x, y, z) \in \mathbb{R}^{3} ; x y=0\right\}$ (double crossing); $\mathcal{T}=\{(x, y, z) \in$ $\left.\mathbb{R}^{3} ; x y z=0\right\}$ (triple crossing); $\mathcal{C}=\left\{(x, y, z) \in \mathbb{R}^{3} ; z^{2}-x^{2}-y^{2}=0\right\}$ (cone) or $\mathcal{W}=\left\{(x, y, z) \in \mathbb{R}^{3} ; z x^{2}-y^{2}=0\right\}$ (Whitney's umbrella).
\end{abstract}

\section{INTRODUCTION}

Non-smooth differential systems appear in applications of various nature: control theory ([13]), systems with hysteresis ([14]) and in mechanical systems with impacts $([3])$. In recent papers (see $[2,9,10,11]$ ) we have established a strong connection between the study of non-smooth systems and the techniques of singular perturbation (for an introducion to the tools of singular perturbation we recommend $[5,6])$.

In this article we are considering systems presenting discontinuities on algebraic varieties. More precisely, let $p_{0} \in \mathcal{U} \subset \mathbb{R}^{3}$ be an open set and $H$ : $\mathcal{U} \rightarrow \mathbb{R}$ a polynomial function. Suppose $p_{0} \in \Sigma=H^{-1}(0), \Sigma^{+}=H^{-1}(0, \infty)$

2010 Mathematics Subject Classification. Primary 34C20, 34C26, 34D15, 34H05.

Key words and phrases. Regularization, vector fields, singular perturbation, nonsmooth vector fields, sliding vector fields, manifolds with simple singularities.

* The first author is partially supported by the MICIIN/FEDER grant MTM200803437, the Generalitat de Catalunya grant 2009SGR-410 and ICREA Academia. The second author and the third authors were partially supported by CAPES, CNPq and FAPESP. All the authors are supported by FP7-PEOPLE-2012-IRSES-316338. 


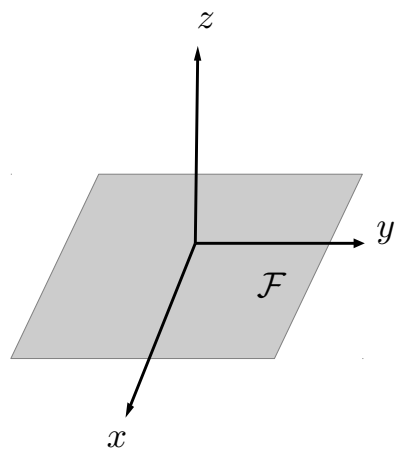

FIGURE 1. Regular switching manifold $H(x, y, z)=0$.

and $\Sigma^{-}=H^{-1}(-\infty, 0)$. Consider the differential system

$$
\dot{p}=X(p)=\left\{\begin{array}{lll}
X_{+}(p), & \text { if } & p \in \Sigma_{+} \\
X_{-}(p), & \text { if } & p \in \Sigma_{-}
\end{array}\right.
$$

with $p=(x, y, z) \in(\mathcal{U} \backslash \Sigma) \subset \mathbb{R}^{3}$, and $X_{+}, X_{-}: \mathcal{U} \rightarrow \mathbb{R}^{3}$ smooth vector fields. The space state $(\mathcal{U} \backslash \Sigma)$ is split into two subsets $\Sigma_{+}$and $\Sigma_{-}$by $\Sigma$. We refer $\Sigma$ as switching manifold and we suppose that there exists $C^{\infty}$ diffeomorphism $\Psi: \mathcal{S} \rightarrow H^{-1}(0), \Psi(0,0,0)=p_{0}$, where $\mathcal{S}$ is one of the following subsets of $\mathbb{R}^{3}$.

(a) $\mathcal{F}=\left\{(x, y, z) \in \mathbb{R}^{3} ; z=0\right\}$. In this case we say that $p_{0}$ is a regular discontinuity and we denote $X=\left(X_{+}, X_{-}\right) \in \Omega(\mathcal{U}, \mathcal{F})$.

(b) $\mathcal{D}=\left\{(x, y, z) \in \mathbb{R}^{3} ; x y=0\right\}$. In this case we say that $p_{0}$ is a double discontinuity and we denote $X=\left(X_{+}, X_{-}\right) \in \Omega(\mathcal{U}, \mathcal{D})$.

(c) $\mathcal{T}=\left\{(x, y, z) \in \mathbb{R}^{3} ; x y z=0\right\}$. In this case we say that $p_{0}$ is a triple discontinuity and we denote $X=\left(X_{+}, X_{-}\right) \in \Omega(\mathcal{U}, \mathcal{T})$.

(d) $\mathcal{C}=\left\{(x, y, z) \in \mathbb{R}^{3} ; z^{2}-x^{2}-y^{2}=0\right\}$. In this case we say that $p_{0}$ is a cone discontinuity and we denote $X=\left(X_{+}, X_{-}\right) \in \Omega(\mathcal{U}, \mathcal{C})$.

(e) $\mathcal{W}=\left\{(x, y, z) \in \mathbb{R}^{3} ; z x^{2}-y^{2}=0\right\}$. In this case we say that $p_{0}$ is a Whitney discontinuity and we denote $X=\left(X_{+}, X_{-}\right) \in \Omega(\mathcal{U}, \mathcal{W})$.

We denote $\varphi_{p}^{+}(t)$, the trajectory of $X_{+}$by $p \in \Sigma_{+}$i.e. :

$$
\frac{d}{d t} \varphi_{p}^{+}(t)=X_{+}\left(\varphi_{p}^{+}(t)\right), \quad \varphi_{p}^{+}(0)=p \in \Sigma_{+} .
$$

Analogously, the trajectory of $X_{-}$by $p \in \Sigma_{-}$is denoted by $\varphi_{p}^{-}(t)$.

The most important and interesting case is when for any initial condition near $p \in H^{-1}(0)$ the corresponding solution trajectories are attracted to $H^{-1}(0)$. In this case, a trajectory which arrives at $H^{-1}(0)$ is forced to remain 

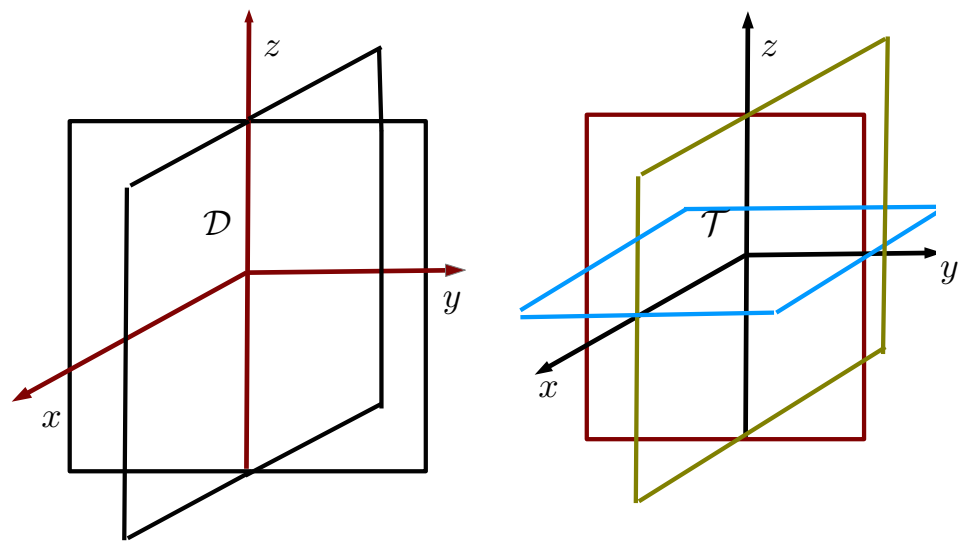

Figure 2. Double (left) and triple (right) crossing switching manifolds.
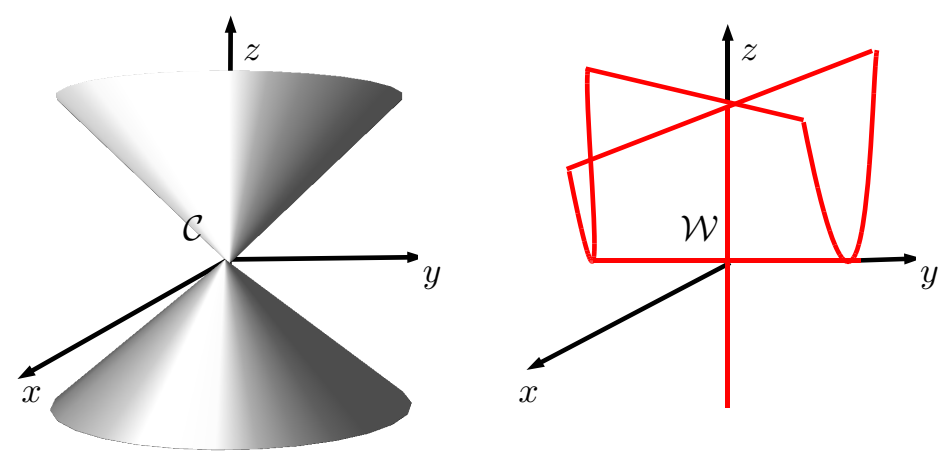

Figure 3. Cone (left) and Whitney's umbrelas (right) crossing switching manifold.

on $H^{-1}(0)$. Filippov ( see [7]) realized that in this situation the motion continues on $\Sigma=H^{-1}(0)$, for the case (a). Without loss of generality we can suppose that $H(x, y, z)=z, \Sigma=\{(x, y, z) \in \mathcal{U}: z=0\}, \Sigma_{+}=\{(x, y, z) \in$ $\mathcal{U}: z>0\}$ and $\Sigma_{-}=\{(x, y, z) \in \mathcal{U}: z<0\}$. Denote $X_{+}=\left(f_{1}, g_{1}, h_{1}\right)$ and $X_{-}=\left(f_{2}, g_{2}, h_{2}\right)$. Following the terminology of [15], the following regions in $\Sigma$ are classified as:

- Sliding Region: $\Sigma^{s l}=\left\{p \in \Sigma: h_{1}(p)<0, h_{2}(p)>0\right\}$. In this case any orbit which meets $\Sigma^{s l}$ remains tangent to $\Sigma$ for positive time. 


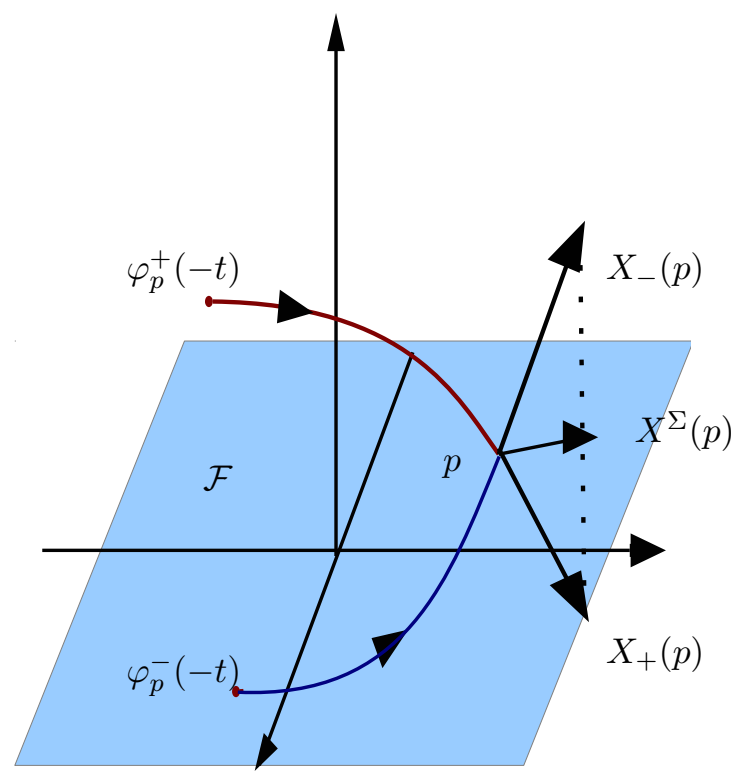

FiguRE 4. Sliding vector field for the regular case.

- Escaping Region: $\Sigma^{e s}=\left\{p \in \Sigma: h_{1}(p)>0, h_{2}(p)<0\right\}$. In this case any orbit which meets $\Sigma^{e s}$ remains tangent to $\Sigma$ for negative time.

- Sewing Region: $\Sigma^{s w}=\left\{p \in \Sigma: h_{1}(p) h_{2}(p)>0\right\}$.

On $\Sigma^{s l} \cup \Sigma^{e s}$ the flow slides on $\Sigma$; it follows a well defined vector field $X^{\Sigma}$ called sliding vector field. The sliding vector field associated with $X=$ $\left(X_{+}, X_{-}\right)$is the vector field $X^{\Sigma}$ tangent to $\Sigma$ and defined by

$$
X^{\Sigma}=\frac{1}{h_{1}-h_{2}}\left(h_{1} f_{2}-h_{2} f_{1}, h_{1} g_{2}-h_{2} g_{1}\right) .
$$

Natural questions appear:

Does the motion continue on $\Sigma=H^{-1}(0)$ for the cases (b), ..., (e)? Is it possible, inside the Filippov convention, to define a sliding vector field on the intersecting switching manifold?

These questions are considered in [4] where a construction for selecting a filippov vector field is done. Here, our approach combines $\varepsilon$-regularization process, blowing-up method and singular perturbation theory. 
Let $X \in \Omega(\mathcal{U}, \Sigma)$ be a non-smooth vector field where $p_{0} \in \mathcal{U} \subset \mathbb{R}^{3}$ is an open set. If $\phi:(\overline{\mathcal{U}}, 0) \rightarrow\left(\mathcal{U}, p_{0}\right)$ is a smooth map we denote $\bar{X}$ the $\phi$-induced vector field which is determined by $\overline{X_{+}}$and $\overline{X_{-}}$on $\phi^{-1}\left(\Sigma_{+}\right)$and on $\phi^{-1}\left(\Sigma_{-}\right)$, respectively. Moreover we denote $\bar{\Sigma}=\partial \phi^{-1}\left(\Sigma_{+}\right) \cup \partial \phi^{-1}\left(\Sigma_{-}\right)$. Our main result is the following.

Theorem 1.1 (Regular sliding). Suppose that $\Sigma=\mathcal{F}$. Then there exists a singular perturbation problem

$$
\dot{x}=\beta(x, y, \theta, r), \quad \dot{y}=\gamma(x, y, \theta, r), \quad r \dot{\theta}=\alpha(x, y, \theta, r)
$$

with $x, y \in \mathbb{R}, \quad \theta \in(0, \pi), \quad r \geq 0$, such that the slow manifold $\mathcal{S}=$ $\{(x, y, \theta) \in \mathbb{R} \times \mathbb{R} \times(0, \pi): \alpha(x, y, \theta, 0)=0\}$ and $\Sigma^{s l} \bigcup \Sigma^{e s}$ are homeomorphic and the reduced problem obtained considering $r=0$ in (2) and the dynamical system corresponding to the sliding vector field (1) are topologically equivalent.

The geometric interpretation of Theorem 1.1 is as follows. By means a polar blow up we may replace the discontinuity $\Sigma$ by the cylinder $\mathbb{R}^{2} \times(0, \pi)$. In this cylinder we draw the phase portrait of the fast-slow system (2), which is composed by a slow manifold and a horizontal fast flow. On the slow manifold we have the phase portrait of the reduced system. The projection of the slow manifold on the surface $\Sigma$ coincides with the sliding region and the reduced system has the same phase portrait as the sliding system. In Section 2 we illustrate this result.

Theorem 1.2 (Double sliding). Suppose that $\Sigma=\mathcal{D}$. The map $\phi:(0,2 \pi) \times$ $[0,+\infty) \times \mathbb{R} \rightarrow \mathbb{R}^{3}$ given by $\phi(\theta, r, z)=(r \cos \theta, r \sin \theta, z)$ induces a vector field $\bar{X}$ satisfying that any discontinuity $q \in \bar{\Sigma}$ is regular.

Theorem 1.3 (Triple sliding). Suppose that $\Sigma=\mathcal{T}$. The map $\phi:(0, \pi) \times$ $(0,2 \pi) \times[0,+\infty) \rightarrow \mathbb{R}^{3}$ given by

$$
\phi(q)=\phi\left(\theta_{1}, \theta_{2}, r\right)=\left(r \sin \theta_{1} \cos \theta_{2}, r \sin \theta_{1} \sin \theta_{2}, r \cos \theta_{1}\right)
$$

induces a non-smooth vector field $\bar{X}$ satisfying that any discontinuity $q \in \bar{\Sigma}$ is either regular or a double crossing.

Theorem 1.4 (Cone sliding). Suppose that $\Sigma=\mathcal{C}$. The map $\phi:(0, \pi) \times$ $(0,2 \pi) \times[0,+\infty) \rightarrow \mathbb{R}^{3}$ given in Theorem 1.3 induces a non-smooth vector field $\bar{X}$ satisfying that any discontinuity $q \in \bar{\Sigma}$ with $\phi(q) \neq 0$ is regular. Moreover the switching manifold on $(0, \pi) \times(0,2 \pi) \times\{0\}$ is homeomorphic to two non-intersecting circles.

Theorem 1.5 (Whitney sliding). Suppose that $\Sigma=\mathcal{W}$. The map $\phi: \mathbb{R} \backslash$ $\{0\} \times \mathbb{R} \times \mathbb{R} \rightarrow \mathbb{R}^{3}$ given by

$$
\phi(u, v, w)=(u, u v, w)
$$


induces a non-smooth vector field $\bar{X}$ satisfying that any discontinuity $q \in \bar{\Sigma}$ with $u \neq 0$ is regular. Moreover, if $q \in \bar{\Sigma}$ is a discontinuity with $u^{2}+w^{2}=0$ then $q$ is a double crossing.

Note that for each double, triple, cone or Whitney descontinuity, we are able to, after a finite number of blow ups, reduce the study to the regular case. Consequently we have a sliding vector field well defined. The basic objective behind these new coordinates is the ability to perform a systematic analysis of the phase portrait of a class of non-smooth systems via singular perturbation tools.

The proofs of our main results are given in Sections 2, 3, 4, 5 and 6 where we study the sliding motion on $\mathcal{F}, \mathcal{D}, \mathcal{T}, \mathcal{C}$ and $\mathcal{W}$ respectively. The sliding vector field idealized by Filippov can not be uniquely extended for intersecting switching discontinuous manifold. However, the blow up method developed here can be applied for such kind of surface. Since this method produces a differential system which is equivalent to the sliding vector field for the regular case, it can be considered as generalization of the Filippov convention. A sufficient number of blow ups to get a sliding vector field like the idealized by Filippov is given in Table 1.

\begin{tabular}{|c||c|}
\hline & $\sharp$ Blow-ups \\
\hline$\Omega(\mathcal{U}, \mathcal{F})$ & 0 \\
\hline$\Omega(\mathcal{U}, \mathcal{D})$ & 1 \\
\hline$\Omega(\mathcal{U}, \mathcal{C})$ & 1 \\
\hline$\Omega(\mathcal{U}, \mathcal{T})$ & 2 \\
\hline$\Omega(\mathcal{U}, \mathcal{W})$ & 2 \\
\hline
\end{tabular}

TABLE 1. Sufficient number of blow ups to get a sliding vector field like the idealized by Filippov.

\section{Proof of Theorem 1.1 - Sliding motion on $\mathcal{F}$}

In this section we assume that $\Sigma=\{(x, y, 0): x, y \in \mathbb{R}\}$ and $X_{+}=$ $\left(f_{1}, g_{1}, h_{1}\right), X_{-}=\left(f_{2}, g_{2}, h_{2}\right)$.

First of all we introduce two basic tools which will be used in the proof.

- A smooth function $\varphi: \mathbb{R} \longrightarrow \mathbb{R}$ is a transition function if $\lim _{s \rightarrow \pm \infty} \varphi(s)=$ \pm 1 and $\varphi^{\prime}(s)>0$ for any $s \in \mathbb{R}$.

- The $\varphi$-regularization of $X=\left(X_{+}, X_{-}\right)$is the one-parameter family $X_{\varepsilon}$ given by

$$
X_{\varepsilon}(x, y, z)=\left(\frac{1}{2}+\frac{\varphi(z / \varepsilon)}{2}\right) X_{+}(q)+\left(\frac{1}{2}-\frac{\varphi(z / \varepsilon)}{2}\right) X_{-}(q),
$$


for $\varepsilon>0$.

It is easy to see that $\psi:[0, \pi] \longrightarrow \mathbb{R}$ given by $\psi(\theta)=\varphi(\cot \theta)$ for $0<\theta<\pi$, $\psi(0)=1$ and $\psi(\pi)=-1$ is a non increasing smooth function.

Proof of Theorem 1.1. We apply the regularization process and then we get that the trajectories of the regularized vector field $X_{\varepsilon}$ are the solutions of the differential system

$$
(\dot{x}, \dot{y}, \dot{z})=\frac{\left(f_{1}+f_{2}, g_{1}+g_{2}, h_{1}+h_{2}\right)+\varphi(z / \varepsilon)\left(f_{1}-f_{2}, g_{1}-g_{2}, h_{1}-h_{2}\right)}{2}
$$

Next we consider the polar blow up coordinates given by $z=r \cos \theta$ and $\varepsilon=r \sin \theta$, with $r \geq 0$ and $\theta \in[0, \pi]$. Using these coordinates the parameter value $\varepsilon=0$ is represented by $r=0$ and the blow up induces the differential system given by

$$
\begin{aligned}
\dot{x} & =\beta(x, y, \theta, r)=\left(\frac{f_{1}+f_{2}}{2}+\psi(\theta) \frac{f_{1}-f_{2}}{2}\right), \\
\dot{y} & =\gamma(x, y, \theta, r)=\left(\frac{g_{1}+g_{2}}{2}+\psi(\theta) \frac{g_{1}-g_{2}}{2}\right), \\
r \dot{\theta} & =\alpha(x, y, \theta, r)=-\sin \theta\left(\frac{h_{1}+h_{2}}{2}+\psi(\theta) \frac{h_{1}-h_{2}}{2}\right) .
\end{aligned}
$$

It is a typical singular perturbation problem. The fast system is the system after a time re-scaling (multiply by $r$ the right hand side of the system) and considering $r=0$ :

$$
x^{\prime}=0, \quad y^{\prime}=0, \quad \theta^{\prime}=\alpha(x, y, \theta, 0) .
$$

The slow flow is given by the solutions of the reduced problem represented by

$$
0=\alpha(x, y, \theta, 0), \quad \dot{x}=\beta(x, y, \theta, 0), \quad \dot{y}=\gamma(x, y, \theta, 0) .
$$

The slow manifold for $\theta \in(0, \pi)$ is implicitly determined by the equation

$$
\alpha=\frac{h_{1}+h_{2}}{2}+\psi(\theta) \frac{h_{1}-h_{2}}{2}=0 .
$$

We have that $-1 \leq-\frac{h_{1}+h_{2}}{h_{1}-h_{2}} \leq 1$, for all $(x, y, 0) \in \Sigma^{s l} \cup \Sigma^{e s}$. Since $\psi^{-1}$ is increasing on $(-1,1)$, the equation $\alpha=0$ defines a continuous graphic.

According with the definition of $X^{\Sigma}$ we have

$$
X^{\Sigma}=\frac{1}{h_{1}-h_{2}}\left(h_{1} f_{2}-h_{2} f_{1}, h_{1} g_{2}-h_{2} g_{1}, 0\right)
$$

The reduced problem is represented by

$$
\dot{x}=\frac{f_{1}+f_{2}}{2}+\psi(\theta) \frac{f_{1}-f_{2}}{2}, \quad \dot{y}=\frac{g_{1}+g_{2}}{2}+\psi(\theta) \frac{g_{1}-g_{2}}{2}
$$


with the restriction given by $\psi(\theta)=-\frac{h_{1}+h_{2}}{h_{1}-h_{2}}$. It follows immediately that the flows of $X^{\Sigma}$ and the reduced problem are equivalent.

Remark about two-dimensional non-smooth dynamical systems. Let $(0,0) \in \mathcal{U} \subseteq \mathbb{R}^{2}$ be an open set. Consider $X=\left(X_{+}, X_{-}\right)$with $X_{+}, X_{-}$: $\mathcal{U} \rightarrow \mathbb{R}^{2}$ smooth vector fields and a switching manifold $(0,0) \in \Sigma=H^{-1}(0)$ where $H: \mathcal{U} \rightarrow \mathbb{R}$ is smooth. We denote $X \in \Omega\left(\mathcal{U}, \mathcal{F}_{2}\right)$ if $\Sigma$ is locally diffeomorphic to $\mathcal{F}_{2}=\{(x, 0): x \in \mathbb{R}\}$. We also establish a connection between singular perturbation problems and the sliding vector fields. More specifically there exists a singular perturbation problem

$$
\dot{x}=\beta(x, \theta, r), \quad r \dot{\theta}=\alpha(x, \theta, r)
$$

with $x \in \mathbb{R}, \quad \theta \in(0, \pi), \quad r \geq 0$, such that the slow manifold $\mathcal{S}=\{(x, \theta) \in$ $\mathbb{R} \times(0, \pi): \alpha(x, \theta, 0)=0\}$ and $\Sigma^{s l} \cup \Sigma^{e s}$ are homeomorphic and the reduced problem obtained considering $r=0$ and the dynamical system corresponding to the sliding vector field (1) are topologically equivalent. This is a particular case of a general study for $n$-dimensional non-smooth dynamical systems and their connection with singular perturbation ( see [10]).

Example. ( T-singularity) We say that $q \in \Sigma$ is a codimension 0 singular point of $\left(X_{+}, X_{-}\right)$of the fold-fold kind if

$$
h_{i}(q)=0, \quad\left(f_{i} \frac{\partial h_{i}}{\partial x}+g_{i} \frac{\partial h_{i}}{\partial y}+h_{i} \frac{\partial h_{i}}{\partial z}\right)(q) \neq 0, \quad i=1,2
$$

and the curves $h_{1}(x, y, 0)=h_{2}(x, y, 0)=0$ along which $X_{+}$and $X_{-}$has quadratic contact with $\Sigma$, are transverse at $q$. Besides, when

$$
\left(f_{1} \frac{\partial h_{1}}{\partial x}+g_{1} \frac{\partial h_{1}}{\partial y}+h_{1} \frac{\partial h_{1}}{\partial z}\right)(q)<0, \quad\left(f_{2} \frac{\partial h_{2}}{\partial x}+g_{2} \frac{\partial h_{2}}{\partial y}+h_{2} \frac{\partial h_{2}}{\partial z}\right)(q)>0
$$

we say that $q$ is a $\boldsymbol{T}$-singularity.

Assume $X_{+}=\left(k_{1}, a, x\right)$ and $X_{-}=\left(b, k_{2}, y\right)$. If $k_{1}<0, k_{2}>0, a+b<0$, $a<b$ and $a b<k_{1} k_{2}$, then $(0,0)$ is a T-singularity.

The regularization $X_{\varepsilon}$ is given by

$$
\begin{gathered}
X_{\varepsilon}=\left(\frac{1}{2}+\frac{\varphi\left(\frac{z}{\varepsilon}\right)}{2}\right) X_{+}+\left(\frac{1}{2}-\frac{\varphi\left(\frac{z}{\varepsilon}\right)}{2}\right) X_{-}= \\
\left(\frac{k_{1}+b}{2}+\varphi\left(\frac{z}{\varepsilon}\right) \frac{k_{1}-b}{2}, \frac{a+k_{2}}{2}+\varphi\left(\frac{z}{\varepsilon}\right) \frac{a-k_{2}}{2}, \frac{x+y}{2}+\varphi\left(\frac{z}{\varepsilon}\right) \frac{x-y}{2}\right) .
\end{gathered}
$$

Performing the blow up $z=r \cos \theta, \varepsilon=r \sin \theta$, where $r \geq 0$ and $\theta \in[0, \pi]$ we get the following singular perturbation problem :

$$
\begin{aligned}
\dot{x} & =\left(k_{1}+b\right) / 2+\varphi(\cot \theta)\left(k_{1}-b\right) / 2, \\
\dot{y} & =\left(a+k_{2}\right) / 2+\varphi(\cot \theta)\left(a-k_{2}\right) / 2, \\
r \dot{\theta} & =-\sin \theta((x+y) / 2+\varphi(\cot \theta)(x-y) / 2) .
\end{aligned}
$$


The slow manifold is represented by

$$
\left\{(x, y, \theta) \in \mathbb{R}^{2} \times(0, \pi):(x+y) / 2+\varphi(\cot \theta)(x-y) / 2=0\right\} .
$$

The graphic of this surface has a helicoidal shape and its intersections with the planes $\theta=$ constant are straight lines. In fact, for $\theta=0$ the line is $x=0$ and for $\theta=\pi$ the line is $y=0$. Moreover for $\theta \in(0, \pi)$ the points $(\theta, x, y)$ satisfying $\varphi(\cot \theta)=-\frac{x+y}{x-y}$ are on a straight line. The slow flow is given by

$$
\left(\begin{array}{c}
\dot{x} \\
\dot{y}
\end{array}\right)=\frac{1}{y-x}\left(\begin{array}{cc}
-b & k_{1} \\
-k_{2} & a
\end{array}\right)\left(\begin{array}{l}
x \\
y
\end{array}\right) .
$$

Since $y-x>0$ in the sliding region $(x<0$ and $y>0)$ it is enough to compute the eigenvalues of the above matrix.

Since $k_{1}<0, k_{2}>0, a-b<0$, and $a b<k_{1} k_{2}$ the eigenvalues

$$
\lambda_{ \pm}=\frac{a-b \pm \sqrt{(b+a)^{2}-4 k_{1} k_{2}}}{2}
$$

are negative. In fact, $-4 k_{1} k_{2}>0$ and then $(b+a)^{2}-4 k_{1} k_{2}>0$. It implies that the eigenvalues are real. Besides,

$$
a b<k_{1} k_{2} \Rightarrow(b+a)^{2}-4 k_{1} k_{2}<(b-a)^{2} .
$$

Since $(a-b)<0$ and $\sqrt{(b+a)^{2}-4 k_{1} k_{2}}<(b-a)=|a-b|$ the eigenvalues are negative. The corresponding eigenvectors are

$$
\omega_{ \pm}=\left(\frac{2 k_{1}}{a+b \pm \sqrt{(a+b)^{2}-4 k_{1} k_{2}}}, 1\right) .
$$

The eigenvector $\omega_{+}$( resp. are $\omega_{-}$) is contained in the sewing (resp. sliding) region. Since $\left|\lambda_{-}\right|>\left|\lambda_{+}\right|$we have that all trajectories (except those ones on the straight line corresponding to $\omega_{-}$) tend to the origin tangentially to the straight line generated by $\omega_{+}$.

\section{Proof of Theorem 1.2 - Sliding motion on $\mathcal{D}$}

In this section we assume that $\Sigma=\left\{(x, y, z) \in \mathbb{R}^{3}: x y=0\right\}, X_{+}=$ $\left(f_{1}, g_{1}, h_{1}\right)$ and $X_{-}=\left(f_{2}, g_{2}, h_{2}\right)$, and $f_{i}, g_{i}$ and $h_{i}$ are $C^{r}$ functions, where $i=1,2$. Moreover we assume that $f_{i}(p)=g_{i}(p)=h_{i}(p)=0$ for any $p \in \Sigma$. In fact, if it is not true, we then consider the vector field

$$
Y(x, y)=\left(x^{2}+y^{2}\right) X(x, y) \text {. }
$$

instead.

Observe that if $p=(x, y, z) \in \Sigma$ is such that $x^{2}+y^{2} \neq 0$ then there exists an open neighborhood $\mathcal{U}_{p} \subset \mathbb{R}^{3}$ of $p$ such that $\left.X\right|_{\mathcal{U}_{p}} \in \Omega\left(\mathcal{U}_{p}, \mathcal{F}\right)$.

Proof of Theorem 1.2. Consider the map $\phi:(0,2 \pi) \times[0,+\infty) \times \mathbb{R} \rightarrow \mathbb{R}^{3}$ given by $\phi(\theta, r, z)=(r \cos \theta, r \sin \theta, z)$. Let $\bar{X}$ be the vector field determined by $\overline{X_{+}}$and $\overline{X_{-}}$on $\phi^{-1}\left(\Sigma_{+}\right)$and on $\phi^{-1}\left(\Sigma_{-}\right)$, respectively, with switching 


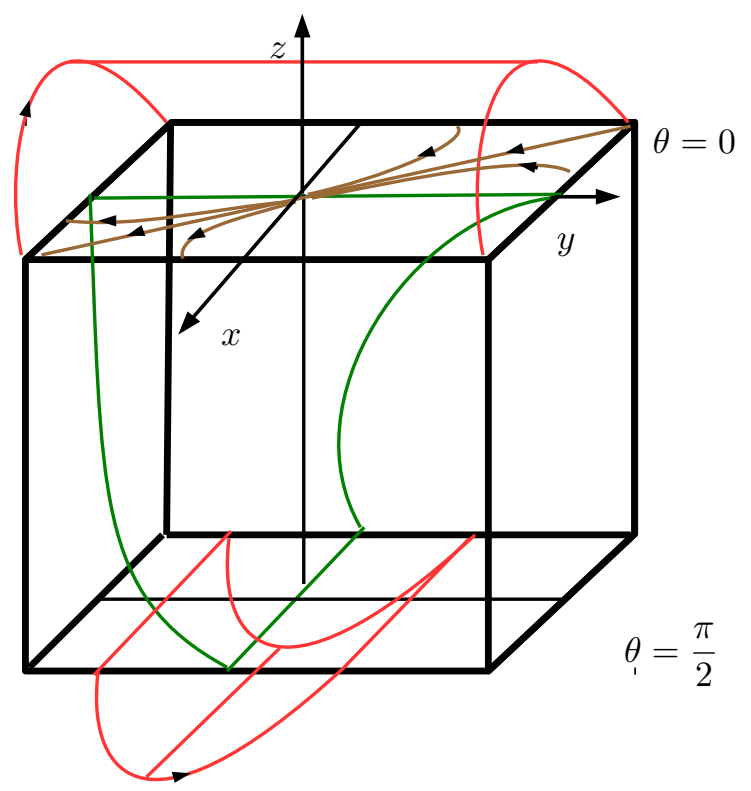

Figure 5. T-singularity. The brown flow is the projection of the slow flow on $\theta=0$.

manifold $\bar{\Sigma}=\partial \phi^{-1}\left(\Sigma_{+}\right) \cup \partial \phi^{-1}\left(\Sigma_{-}\right)$.

1) If $r>0$ and $q=(\theta, r, z) \in \bar{\Sigma}$ then there exists a neighborhood $\mathcal{U}_{q} \subset S^{1} \times[0,+\infty) \times \mathbb{R}$ such that $\left.\bar{X}\right|_{\mathcal{U}_{q}} \in \Omega\left(\mathcal{U}_{q}, \mathcal{F}\right)$. In fact, for $r>0$ we have that $\phi^{\prime}(\theta, r, z)$ is an isomorphism. It implies that the gradient $\nabla(F \circ \phi) \neq 0$ on $(F \circ \phi)^{-1}(0) \cap\{r>0\}$.

2) If $q=(\theta, 0, z) \in \bar{\Sigma}$ then there exists a neighborhood $\mathcal{U}_{q} \subset S^{1} \times \mathbb{R}$ such that $\left.\bar{X}\right|_{\mathcal{U}_{q}} \in \Omega\left(\mathcal{U}_{q}, \mathcal{F}_{2}\right)$. For $r=0$ we have

$$
\bar{\Sigma} \cap\{r=0\}=G^{-1}(0)
$$

with $G(\theta, r, z)=\theta(\theta-\pi / 2)(\theta-\pi)(\theta-3 \pi / 2)$. Besides it is easy to see that $\nabla G(\theta, 0, z) \neq 0$ on $G^{-1}(0)$.

Remark. The vector field $\bar{X}$ determined by $\overline{X_{+}}$and $\overline{X_{-}}$and induced by $\phi$ on $(0, \pi) \times[0,+\infty) \times \mathbb{R}$ satisfies

$$
r \dot{\theta}=g_{i} \cos \theta-f_{i} \sin \theta, \quad \dot{r}=f_{i} \cos \theta+g_{i} \sin \theta, \quad \dot{z}=h_{i} .
$$




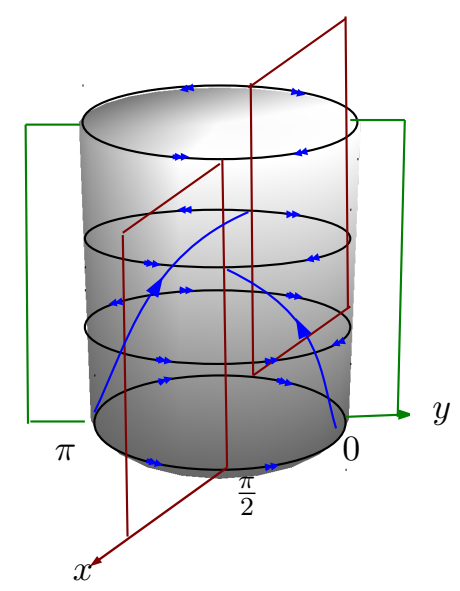

Figure 6 . Non-smooth systems on the cylinder $S^{1} \times \mathbb{R}$.

Considering $r=0$ and eliminating common factors, we get the reduced flow

$$
0=g_{i} \cos \theta-f_{i} \sin \theta, \quad \dot{z}=h_{i} .
$$

There exists a sliding curve on the cylinder $S^{1} \times \mathbb{R}$ defined implicitly by the equations

$$
\begin{aligned}
& 0=g_{1} \cos \theta-f_{1} \sin \theta, \quad \theta \in\left(0, \frac{\pi}{2}\right) \cup\left(\pi, \frac{3 \pi}{2}\right), \\
& 0=g_{2} \cos \theta-f_{2} \sin \theta, \quad \theta \in\left(\frac{\pi}{2}, \pi\right) \cup\left(\frac{3 \pi}{2}, 2 \pi\right) .
\end{aligned}
$$

Example . Consider the non-smooth vector field $X=\left(X_{+}, X_{-}\right)$given by $X_{+}(x, y, z)=(-z, 1,1)$ for $x y>0$ and $X_{-}(x, y, z)=(1,1,-1)$ for $x y<0$. If $p=(x, y, z) \in \Sigma$ is such that $y=0, x \neq 0$ or $x=0, y \neq 0$ then there exists an open neighborhood $\mathcal{U}_{p} \subseteq \mathbb{R}^{3}$ of $p$ such that $\left.X\right|_{\mathcal{U}_{p}} \in \Omega\left(\mathcal{U}_{p}, \mathcal{F}\right)$. If $x=y=0$ then there exists an open neighborhood $\mathcal{U}_{p} \subseteq \mathbb{R}^{3}$ of $p$ such that $\left.X\right|_{\mathcal{U}_{p}} \in \Omega\left(\mathcal{U}_{p}, \mathcal{D}\right)$. Applying Theorem 1.2 the equations of the non-smooth system on the cylinder $[0,2 \pi] \times \mathbb{R}$ are

$$
\eta \dot{\theta}=\cos \theta+z \sin \theta, \quad \dot{z}=1
$$

for $\theta \in(0, \pi / 2) \cup(\pi, 3 \pi / 2)$ and

$$
\eta \dot{\theta}=\cos \theta-\sin \theta, \quad \dot{z}=-1
$$

for $\theta \in(\pi / 2, \pi) \cup(3 \pi / 2,2 \pi)$.

\section{Proof Theorem 1.3 - Sliding motion on $\mathcal{T}$}

In this section we assume that $\Sigma=\left\{(x, y, z) \in \mathbb{R}^{3}: x y z=0\right\}, X_{+}=$ $\left(f_{1}, g_{1}, h_{1}\right)$ and $X_{-}=\left(f_{2}, g_{2}, h_{2}\right)$. 
We denote $L_{x}=\{y=z=0\}, L_{y}=\{x=z=0\}$ and $L_{z}=\{x=y=0\}$. For $p=(x, y, z) \in \Sigma$ we have

- If $p \in \Sigma \backslash\left(L_{x} \cup L_{y} \cup L_{z}\right)$ then then there exists an open neighborhood $\mathcal{U}_{p} \subset \mathbb{R}^{3}$ of $p$ such that $\left.X\right|_{\mathcal{U}_{p}} \in \Omega\left(\mathcal{U}_{p}, \mathcal{F}\right)$.

- If $p \in\left(L_{x} \cup L_{y} \cup L_{z}\right)$ but $p \neq 0$ then then there exists an open neighborhood $\mathcal{U}_{p} \subset \mathbb{R}^{3}$ of $p$ such that $\left.X\right|_{\mathcal{U}_{p}} \in \Omega\left(\mathcal{U}_{p}, \mathcal{D}\right)$.

Proof of Theorem 1.3. Consider the map $\phi:(0, \pi) \times(0,2 \pi) \times[0,+\infty) \rightarrow \mathbb{R}^{3}$ given by $\phi\left(\theta_{1}, \theta_{2}, r\right)=\left(r \sin \theta_{1} \cos \theta_{2}, r \sin \theta_{1} \sin \theta_{2}, r \cos \theta_{1}\right)$. Then the vector field $\bar{X}$ determined by $\overline{X_{+}}$and $\overline{X_{-}}$on $\phi^{-1}\left(\Sigma_{+}\right)$and on $\phi^{-1}\left(\Sigma_{-}\right)$, respectively, and induced by $\phi$ satisfies the following.

1) If $r>0$ and $\phi(q) \in\left(\Sigma \backslash\left(L_{x} \cup L_{y} \cup L_{z}\right)\right)$ then there exists a neighborhood $\mathcal{U}_{q} \subset S^{2} \times \mathbb{R}^{+}$such that $\left.\bar{X}\right|_{\mathcal{U}_{q}} \in \Omega\left(\mathcal{U}_{q}, \mathcal{F}\right)$.

2) There are six "half-spaces" where the discontinuities of $\bar{X}$ are double crossing

- $\left\{\theta_{1}=0\right\} \times \mathbb{R}^{+}$,

- $\left\{\theta_{1}=\pi\right\} \times \mathbb{R}^{+}$

- $\left\{\theta_{1}=\frac{\pi}{2}\right.$ and $\left.\theta_{2}=\frac{\pi}{2}\right\} \times \mathbb{R}^{+}$,

- $\left\{\theta_{1}=\frac{\pi}{2}\right.$ and $\left.\theta_{2}=\frac{3 \pi}{2}\right\} \times \mathbb{R}^{+}$

- $\left\{\theta_{1}=\frac{\pi}{2}\right.$ and $\left.\theta_{2}=0\right\} \times \mathbb{R}^{+}$,

- $\left\{\theta_{1}=\frac{\pi}{2}\right.$ and $\left.\theta_{2}=\pi\right\} \times \mathbb{R}^{+}$.

3) If $q \in S^{2} \times\{0\}$ and $\left\{\sin \theta_{2}=0\right\} \cap\left\{\sin \theta_{1} \cos \theta_{1} \neq 0\right\} \cup\left\{\cos \theta_{1}=\right.$ $0\} \cap\left\{\sin \theta_{2} \cos \theta_{2} \neq 0\right\} \cup\left\{\cos \theta_{2}=0\right\} \cap\left\{\sin \theta_{1} \cos \theta_{1} \neq 0\right\}$ then there exists a neighborhood $\mathcal{U}_{q} \subset S^{2} \times\{0\}$ such that $\left.\bar{X}\right|_{\mathcal{U}_{q}} \in \Omega\left(\mathcal{U}_{q}, \mathcal{F}_{2}\right)$. If $q=\left(\theta_{1}, \theta_{2}, 0\right) \in$ $S^{2} \times\{0\}$ is such that

$$
\left(\theta_{1}, \theta_{2}\right) \in\{(0,0),(\pi, 0),(\pi / 2, \pm \pi / 2),(\pi / 2, \pm \pi)\}
$$

then there exists a neighborhood $\mathcal{U}_{q} \subset S^{2} \times\{0\}$ such that $\left.\bar{X}\right|_{\mathcal{U}_{q}} \in \Omega\left(\mathcal{U}_{q}, \mathcal{D}\right)$.

The trajectories of $X_{i}, \quad i=1,2$, are given by the solutions of the differential system

$$
x^{\prime}=f_{i}(x, y, z), \quad y^{\prime}=g_{i}(x, y, z), \quad z^{\prime}=h_{i}(x, y, z) .
$$

The trajectories of the $\phi$ induced vector field $\overline{X_{i}}$ on $S^{2} \times[0,+\infty)$ are the solutions of

$$
\begin{aligned}
\theta_{1}^{\prime} & =f_{i}\left[\frac{\cos \theta_{1} \cos \theta_{2}}{r}\right]+g_{i}\left[\frac{\cos \theta_{1} \sin \theta_{2}}{r}\right]+h_{i}\left[\frac{-\sin \theta_{1}}{r}\right], \\
\theta_{2}^{\prime} & =f_{i}\left[\frac{-\sin \theta_{2}}{r \sin \theta_{1}}\right]+g_{i}\left[\frac{\cos \theta_{2}}{r \sin \theta_{1}}\right], \\
r^{\prime} & =f_{i}\left[\sin \theta_{1} \cos \theta_{2}\right]+g_{+}\left[\sin \theta_{1} \sin \theta_{2}\right]+h_{i}\left[\cos \theta_{1}\right],
\end{aligned}
$$




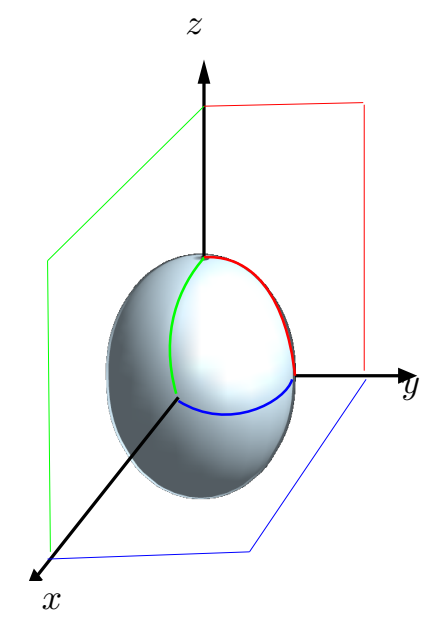

Figure 7. Switching manifold after blow up - case $\mathcal{T}$.

where the functions $f_{i}, g_{i}, h_{i}, i=1,2$, are evaluated at

$$
\left(r \sin \theta_{1} \cos \theta_{2}, r \sin \theta_{1} \sin \theta_{2}, r \cos \theta_{1}\right) .
$$

Since we can assume that $X_{i}(p)=0$ for all $p \in \Sigma$, the system above on $S^{2} \times[0,+\infty)$ becomes

$$
\begin{aligned}
& \theta_{1}^{\prime}=\overline{f_{i}}\left[\sin \theta_{1} \cos \theta_{1} \cos \theta_{2}\right]+\overline{g_{i}}\left[\sin \theta_{1} \cos \theta_{1} \sin \theta_{2}\right]+\overline{h_{i}}\left[-\sin \theta_{1}\right], \\
& \theta_{2}^{\prime}=\overline{f_{i}}\left[-\sin \theta_{2}\right]+\overline{g_{i}}\left[\cos \theta_{2}\right], \\
& r^{\prime}=\overline{f_{i}}\left[r \sin ^{2} \theta_{1} \cos \theta_{2}\right]+\overline{g_{i}}\left[r \sin ^{2} \theta_{1} \sin \theta_{2}\right]+\overline{h_{i}}\left[r \cos \theta_{1}\right],
\end{aligned}
$$

where $\overline{f_{i}}=\lim _{\left(r, \theta_{1}\right) \rightarrow(0,0)} \frac{f_{i}}{r \sin \theta_{1}}, \overline{g_{i}}=\lim _{\left(r, \theta_{1}\right) \rightarrow(0,0)} \frac{g_{i}}{r \sin \theta_{1}}$ and $\overline{h_{i}}=\lim _{r \rightarrow 0} \frac{h_{i}}{r}$.

\section{SLIDing MOtion ON $\mathcal{C}$}

In this section we assume that $\Sigma=\left\{(x, y, z) \in \mathbb{R}^{3}: z^{2}-x^{2}-y^{2}=0\right\}$, $X_{+}=\left(f_{1}, g_{1}, h_{1}\right)$ and $X_{-}=\left(f_{2}, g_{2}, h_{2}\right)$.

If $p \in \Sigma \backslash\{(0,0,0)\}$ then then there exists an open neighborhood $\mathcal{U}_{p} \subset \mathbb{R}^{3}$ of $p$ such that $\left.X\right|_{\mathcal{U}_{p}} \in \Omega\left(\mathcal{U}_{p}, \mathcal{F}\right)$. In fact, $H(x, y, z)=z^{2}-x^{2}-y^{2}$ and $\nabla H(x, y, z)=(-2 x,-2 y, 2 z) \neq(0,0,0)$ for all $(x, y, z) \neq(0,0,0)$.

Proof of Theorem 1.4. Consider the map $\phi:(0, \pi) \times(0,2 \pi) \times[0,+\infty) \rightarrow \mathbb{R}^{3}$ given by $\phi\left(\theta_{1}, \theta_{2}, r\right)=\left(r \sin \theta_{1} \cos \theta_{2}, r \sin \theta_{1} \sin \theta_{2}, r \cos \theta_{1}\right)$. Then the vector 


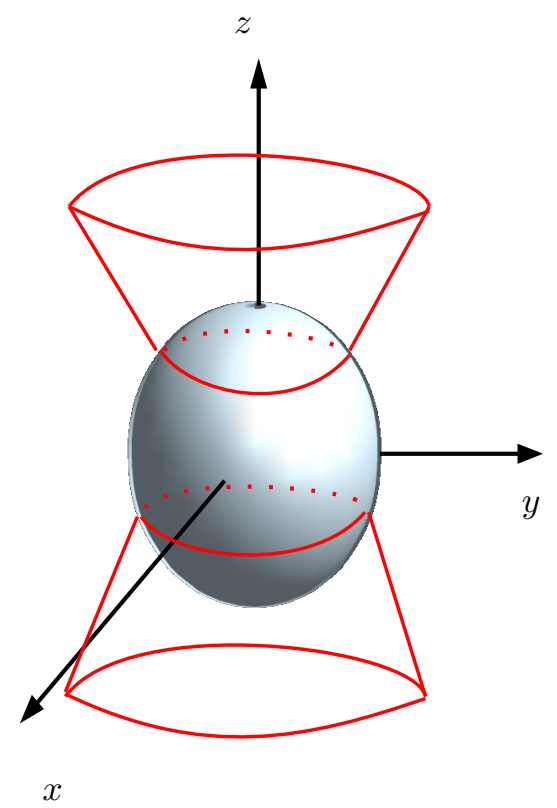

FiguRE 8. Switching manifold after blow up - case $\mathcal{C}$.

field $\bar{X}$ determined by $\overline{X_{+}}$and $\overline{X_{-}}$on $\phi^{-1}\left(\Sigma_{+}\right)$and on $\phi^{-1}\left(\Sigma_{-}\right)$, respectively, and induced by $\phi$ satisfies the following.

1) If $r>0$ then $\phi(q) \in(\Sigma \backslash\{(0,0,0)\}$ and there exists a neighborhood $\mathcal{U}_{q} \subset S^{2} \times \mathbb{R}^{+}$such that $\left.\bar{X}\right|_{\mathcal{U}_{q}} \in \Omega\left(\mathcal{U}_{q}, \mathcal{F}\right)$.

2) If $r=0$ there are two circles $\theta_{1}=\frac{\pi}{4}$ and $\theta_{1}=\frac{3 \pi}{4}$ where the discontinuities of $\bar{X}$ are regular. More precisely, if $q \in S^{2}$ with $\theta_{1}=\frac{\pi}{4}$ or $\theta_{1}=\frac{3 \pi}{4}$ then there exists a neighborhood $\mathcal{U}_{q} \subset S^{2} \times\{0\}$ such that $\left.\bar{X}\right|_{\mathcal{U}_{q}} \in \Omega\left(\mathcal{U}_{q}, \mathcal{F}_{2}\right)$. 
As in Section 4 the trajectories of the $\phi$ induced vector field $\overline{X_{i}}$ on $S^{2} \times$ $[0,+\infty)$ are the solutions of

$$
\begin{aligned}
\theta_{1}^{\prime} & =f_{i}\left[\frac{\cos \theta_{1} \cos \theta_{2}}{r}\right]+g_{i}\left[\frac{\cos \theta_{1} \sin \theta_{2}}{r}\right]+h_{i}\left[\frac{-\sin \theta_{1}}{r}\right], \\
\theta_{2}^{\prime} & =f_{i}\left[\frac{-\sin \theta_{2}}{r \sin \theta_{1}}\right]+g_{i}\left[\frac{\cos \theta_{2}}{r \sin \theta_{1}}\right], \\
r^{\prime} & =f_{i}\left[\sin \theta_{1} \cos \theta_{2}\right]+g_{+}\left[\sin \theta_{1} \sin \theta_{2}\right]+h_{i}\left[\cos \theta_{1}\right],
\end{aligned}
$$

where the functions are evaluated at $\left(r \sin \theta_{1} \cos \theta_{2}, r \sin \theta_{1} \sin \theta_{2}, r \cos \theta_{1}\right)$. Since we can assume that $X_{i}(p)=0$ for all $p \in \Sigma$, the system above on $S^{2} \times[0,+\infty)$ becomes

$$
\begin{aligned}
& \theta_{1}^{\prime}=P_{i}\left(\theta_{1}, \theta_{2}\right)=\overline{f_{i}}\left[\sin \theta_{1} \cos \theta_{1} \cos \theta_{2}\right]+\overline{g_{i}}\left[\sin \theta_{1} \cos \theta_{1} \sin \theta_{2}\right]+\overline{h_{i}}\left[-\sin \theta_{1}\right], \\
& \theta_{2}^{\prime}=Q_{i}\left(\theta_{1}, \theta_{2}\right)=\overline{f_{i}}\left[-\sin \theta_{2}\right]+\overline{g_{i}}\left[\cos \theta_{2}\right],
\end{aligned}
$$

where $\overline{f_{i}}=\lim _{\left(r, \theta_{1}\right) \rightarrow(0,0)} \frac{f_{i}}{r \sin \theta_{1}}, \overline{g_{i}}=\lim _{\left(r, \theta_{1}\right) \rightarrow(0,0)} \frac{g_{i}}{r \sin \theta_{1}}$ and $\overline{h_{i}}=\lim _{r \rightarrow 0} \frac{h_{i}}{r}$.

Consider $\bar{X}^{0}=\left(\bar{X}_{+}^{0}, \bar{X}_{-}^{0}\right)$ the vector field on $S^{2} \times\{0\}$ induced by $\phi$ with discontinuities occuring on the circles $\theta_{1}=\frac{\pi}{4}$ and $\theta_{1}=\frac{3 \pi}{4}$. We denote

$$
\Sigma_{0}^{+}=\left\{\left(\theta_{1}, \theta_{2}\right) ; 0<\theta_{1}<\frac{\pi}{4} \quad \text { or } \quad \frac{3 \pi}{4}<\theta_{1}<\pi\right\}
$$

and

$$
\Sigma_{0}^{-}=\left\{\left(\theta_{1}, \theta_{2}\right) ; \frac{\pi}{4}<\theta_{1}<\frac{3 \pi}{4}\right\} .
$$

The $\varphi$-regularization of $\bar{X}^{0}=\left(\bar{X}_{+}^{0}, \bar{X}_{-}^{0}\right)$ is the one-parameter family $X_{\varepsilon}^{0}$ given by

$$
\bar{X}_{\varepsilon}^{0}=\left(\frac{1}{2}+\frac{\varphi\left(\frac{\theta_{1}-\frac{\pi}{4}}{\varepsilon}\right)}{2}\right) \bar{X}_{+}^{0}+\left(\frac{1}{2}-\frac{\varphi\left(\frac{\theta_{1}-\frac{\pi}{4}}{\varepsilon}\right)}{2}\right) \bar{X}_{-}^{0},
$$

where $\varphi$ is a transition function and $\varepsilon>0$.

The trajectories of the regularized vector field $\bar{X}_{\varepsilon}^{0}$ are the solutions of

$$
\begin{gathered}
\dot{\theta_{1}}=\frac{P_{1}+P_{2}}{2}+\varphi\left(\frac{\theta_{1}-\frac{\pi}{4}}{\varepsilon}\right) \frac{P_{1}-P_{2}}{2}, \\
\dot{\theta_{2}}=\frac{Q_{1}+Q_{2}}{2}+\varphi\left(\frac{\theta_{1}-\frac{\pi}{4}}{\varepsilon}\right) \frac{Q_{1}-Q_{2}}{2} .
\end{gathered}
$$

Next we consider the polar blow up coordinates given by $\theta_{1}-\frac{\pi}{4}=\rho \cos \xi$ and $\varepsilon=\rho \sin \xi$, with $\rho \geq 0$ and $\xi \in[0, \pi]$. Using these coordinates the 
parameter value $\varepsilon=0$ is represented by $\rho=0$ and the blow up induces the differential system given by

$$
\begin{aligned}
\dot{\theta_{2}} & =\beta\left(\theta_{2}, \xi, \rho\right)=\left(\frac{Q_{1}+Q_{2}}{2}+\varphi(\cot \xi) \frac{Q_{1}-Q_{2}}{2}\right), \\
\rho \dot{\xi} & =\gamma\left(\theta_{2}, \xi, \rho\right)=-\sin \xi\left(\frac{P_{1}+P_{2}}{2}+\varphi(\cot \xi) \frac{P_{1}-P_{2}}{2}\right) .
\end{aligned}
$$

We get that the slidding region in the circle $\theta_{1}=\frac{\pi}{4}$ is homeomorphic to the slow manifold

$$
\frac{P_{1}+P_{2}}{2}+\varphi(\cot \xi) \frac{P_{1}-P_{2}}{2}=0
$$

and the slidding vector field is topologicaly equivalent to the slow flow

$$
\begin{aligned}
& \dot{\theta_{2}}=\beta\left(\theta_{2}, \xi, 0\right)=\left(\frac{Q_{1}+Q_{2}}{2}+\varphi(\cot \xi) \frac{Q_{1}-Q_{2}}{2}\right), \\
& 0 \quad=\gamma\left(\theta_{2}, \xi, 0\right)=-\sin \xi\left(\frac{P_{1}+P_{2}}{2}+\varphi(\cot \xi) \frac{P_{1}-P_{2}}{2}\right) .
\end{aligned}
$$

\section{Sliding mOtion on $\mathcal{W}$}

In this section we assume that $\Sigma=\left\{(x, y, z) \in \mathbb{R}^{3}: z x^{2}-y^{2}=0\right\}, X_{+}=$ $\left(f_{1}, g_{1}, h_{1}\right)$ and $X_{-}=\left(f_{2}, g_{2}, h_{2}\right)$. Moreover we assume that $f_{i}(0, y, z)=$ $g_{i}(0, y, z)=0, i=1,2$.

If $p \in \Sigma$ with $x^{2}+y^{2} \neq 0$ then there exists an open neighborhood $\mathcal{U}_{p} \subset \mathbb{R}^{3}$ of $p$ such that $\left.X\right|_{\mathcal{U}_{p}} \in \Omega\left(\mathcal{U}_{p}, \mathcal{F}\right)$.

If $p \in \Sigma$ with $x^{2}+y^{2}=0$ and $z<0$ then we extend smoothly $X$ assuming that $X(p)=X^{+}(p)$.

Proof of Theorem 1.5. Consider the map $\phi: \mathbb{R} \backslash\{0\} \times \mathbb{R} \times \mathbb{R} \rightarrow \mathbb{R}^{3}$ given by $\phi(u, v, w)=(u, u v, w)$. Then the vector field $\bar{X}$ determined by $\overline{X_{+}}$and $\overline{X_{-}}$on $\phi^{-1}\left(\Sigma_{+}\right)$and on $\phi^{-1}\left(\Sigma_{-}\right)$, respectively, and induced by $\phi$ satisfies the following.

a) The discontinuous set is $\Sigma=\left\{(u, v, w) ; u=0 \quad\right.$ or $\left.\quad w=v^{2}\right\}$.

b) If $q \in \Sigma$ with $u \neq 0$ then $q$ is regular.

c) If $q \in \Sigma$ with $u=0$ and $w=v^{2}$ then $q$ is a double singular point. In fact, the surfaces $u=0$ and $w=v^{2}$ are in general position because theirs tangent spaces are transversal

Application. Consider the third order equation

$$
\dddot{x}+a \ddot{x}+b \dot{x}+c x=-\alpha K x
$$




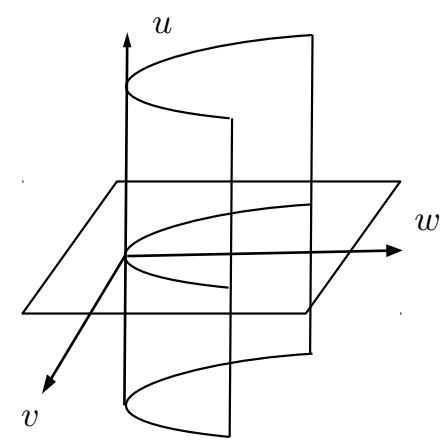

Figure 9. Switching manifold after blow up - case $\mathcal{W}$.

with $a, b, c \in \mathbb{R}, K \in \mathbb{R}^{+}$and $|\alpha| \leq 1$. The corresponding system is

$$
\dot{x}=y, \quad \dot{y}=z, \quad \dot{z}=-c x-b y-a z-\alpha K x .
$$

In [1] the authors study the law of variation of $\alpha$ which ensure asymptotic stability for the zero solution of equation (13). To do this they consider $v(x, y, z)$ a function of the variables $(x, y, z)$ and choose $\alpha$ satisfying that the rate of decrease of $v$ along the trajectory is the gratest. The rate is

$$
\dot{v}=\frac{\partial v}{\partial x} y+\frac{\partial v}{\partial y} z-\frac{\partial v}{\partial z}(c x+b y+a z)-\alpha K \frac{\partial v}{\partial z} x
$$

and it is the greatest by setting

$$
\alpha=\operatorname{sgn} \frac{\partial v}{\partial z} x
$$

Thus the switching manifold is a double crossing

$$
\mathcal{S}=\left\{\frac{\partial v}{\partial z} x=0\right\}
$$

The authors consider $v=C x^{2}+E y^{2}+z^{2}+2 D x y+2 A x z+2 B y z$ and $\alpha=\operatorname{sgn}(A x+B y+z) x$. However if we consider $v=\frac{z^{2}}{2}$ then the switching manifold is given by $x z=0$ which is also a double crossing. Moreover, for $v=\frac{y z^{2}}{2}$ the switching manifold is given by $x y z=0$ which is a triple crossing.

\section{REFERENCES}

[1] Barbashin, E.A. (1970) Introduction to the theory of stability. Translated from the Russian by Transcripta Service, London. Edited by T. Lukes Wolters-Noordhoff Publishing, Groningen. 
[2] Buzzi, C., Silva, P.R. and Teixeira, M.A. (2006). A singular approach to discontinuous vector fields on the plane, J. Diff. Equations 231, 633-655.

[3] di Bernardo, M., Budd, C.J., Champneys, A.R. and Kowalczyk, P. (2008). Piecewisesmooth dynamical systems. Theory and aplications, Applied Mathematical Science 163, Springer-Verlag, London.

[4] Dieci, L., Lopez, L. (2011). Sliding motion on discontinuity surfaces of high codimension. A construction for selecting a Filippov vector field, Numerische Mathematik - NUMER MATH 177-4, 779-811.

[5] Dumortier, F. and Roussarie, R. (1996) Canard cycles and center manifolds, Memoirs Amer. Mat. Soc. 121.

[6] Fenichel, N. (1979). Geometric singular perturbation theory for ordinary differential equations, J. Diff. Equations 31, 53-98.

[7] Filippov, A.F. (1988). Differential equations with discontinuous right-hand sides, Mathematics and its Applications (Soviet Series), Kluwer Academic Publishers, Dordrecht.

[8] Gutierrez, C. and Sotomayor, J. (1982). Stable vector fields on manifolds with simple singularities, Proc. London. Math. Soc 3-45, 97-112.

[9] Llibre, J., Silva, P.R. and Teixeira, M.A. (2007). Regularization of discontinuous vector fields via singular perturbation, J. Dynam. Differential Equation.19, n.2, 309331.

[10] Llibre, J., Silva, P.R. and Teixeira, M.A. (2008). Sliding vector fields via slow fast systems, Bulletin of the Belgian Mathematical Society Simon Stevin 15, 851-869.

[11] Llibre, J., Silva, P.R. and Teixeira, M.A. (2009). Study of singularities in non smooth dynamical systems via singular perturbation, SIAM Journal on Applied Dynamical Systems 8, 508-526.

[12] Llibre, J. and Teixeira, M.A. (1997). Regularization of discontinuous vector fields in dimension three, Discrete Contin. Dynam. Systems 3, 235-241.

[13] Minorsky, N. (1969) Theory of nonlinear control systems. McGraw- 994 Hill, New York, London, Sydney, pp xx+331.

[14] Seidman, T. (2012). Some aspects of modeling with discontinuities, Handbook of evolution equations (Editors: Gaston M. N'Guerekata), Nova Science Pub Inc, New York, pp 311.

[15] Sotomayor, J. and Teixeira, M.A. (1996). Regularization of discontinuous vector fields, International Conference on Differential Equations, Lisboa, Equadiff 95, 207223.

${ }^{1}$ Departament de Matemàtiques, Universitat Autònoma de Barcelona, 08193 Bellaterra, Barcelona, Catalonia, Spain.

2 Departamento de Matemática - IBilce-Unesp, Rua C. Colombo, 2265, CEP 15054-000 S. J. Rio Preto, SÃo Paulo, Brazil

3 IMECC-UNICAMP, CEP 13081-970, CAMPINAS, SÃo PaUlo, Brazil

E-mail address: jllibre@mat.uab.cat

E-mail address: prs@ibilce.unesp.br

E-mail address: teixeira@ime.unicamp.br 